\title{
Building and Evaluating a Mobile Application for an Academic Library
}

\author{
Stan Kurkovsky \\ Department of Computer Science \\ Central Connecticut State University \\ New Britain, CT, USA \\ kurkovskysta@ccsu.edu
}

\author{
Wittawat Meesangnil \\ Elihu Burritt Library, Electronic Resources \& Information Systems \\ Central Connecticut State University \\ New Britain, CT, USA \\ meesangnilwit@ccsu.edu
}

\begin{abstract}
In order to stay relevant to the needs of the modern users, libraries need to make their catalogs and other services easily accessible on mobile devices. Libraries face a number of unique challenges in this process related to 'mobilizing' catalog access, integration third-party information systems, and operating with very limited resources. This paper describes an effort to create a mobile application for an academic library, which includes identifying library services relevant to mobile users, minimizing development costs by utilizing a number of open-source components, and evaluating the usability of the resulting application.
\end{abstract}

Keywords-libraries; mobile information access; Android; usability; user study

\section{INTRODUCTION}

The number of mobile phone users has increased dramatically over the past few years. The current generation of smartphones has become as powerful as desktop computers of just a few years ago. Coupled with anytime anywhere network access, mobile devices have created a paradigm shift where technology became virtually invisible in everyday objects and activities. In response to this trend, many information providers and businesses have embraced this new information access model.

Today, users expect businesses and non-profit organizations not only to have a web presence, but to also provide some services that are especially tailored to the needs of mobile users. Libraries have generally been somewhat late to adopt this trend. Although some perceive libraries as irrelevant in the information society where information is available freely over the Internet, many believe that libraries will not become obsolete, but rather evolve. Libraries will gradually shift from a place where the information is available in a physical format to a new, easy accessibility over the Internet. In order for libraries to become more successful, they must adapt and use new technologies to their maximum potential. Mobile technology is one of the main areas that libraries need to address to provide better and faster services to their users.

This paper describes our efforts to create a mobile application for the library patrons at Central Connecticut State University (CCSU), a large comprehensive Masters level university in the US. We used a community approach to building the application relying on a collection of opensource technologies as a foundation of this project. Such an approach could enable creating a robust application while using very limited resources. The features of this application are limited to those that are relevant to a mobile user. The final outcome of this project is a simple and focused application that reduces the risk of library patron getting lost in the application. The application includes four main components: general information about the library (library hours, phone numbers, directions, and reference services), 'mobilized' library catalog, interactive indoor library map, and a Quick Response (QR) code reader.

This paper is organized as follows. Section II presents related work in the areas of mobile information access, creating mobile library services, trade-offs of creating a native or a web application for a mobile platform, as well as using QR codes in libraries. Section III provides a detailed overview of the system architecture, while Section IV describes the methodology and results of the usability study. Section V concludes the paper with a summary and outlines the directions for future work.

\section{RELATED WORK}

In a typical usage scenario, a library patron would look up the call number on a desktop computer located in the library building or elsewhere, write down, print or email themselves the call numbers, and take that information on paper into the library. In order to retrieve each book, the patron would need to find the corresponding stacks location. Having a mobile application or a mobile website could present a convenient solution to carry both the search capabilities and their results on the same device.

Current research in mobile information access suggests that although browsing dominates web access on mobile devices, users tend to gravitate towards accessing search engines and portals. Mobile search sessions typically last longer and have richer information content compared to mobile web browsing [5]. At the same time, there is a trend for mobile search to move towards the use of search portals, applications or websites offering search capabilities in the context of a specific domain [4]. An on-the-go user typically has an immediate need for information, which would require an easy and simple search method to find that information. This factor further underscores the user trend to seek and use applications for specialized searches [11].

Even though libraries do not appear to be at the forefront of the mobile business ecosystem, library communities are well aware of the rising numbers of mobile devices. The number of mobile devices in general is increasing 
significantly, with the young generation showing the highest rates of technology adoption. A recent study shows that $71 \%$ of young people 12-17 years of age in the USA own a mobile phone [10]. In the education sector, a study released by the EDUCAUSE Center for Applied Research in 2009 found that $51 \%$ of undergraduate respondents owned a webenabled phone and another $12 \%$ planned to purchase one in the next 12 months; $74 \%$ of the students who had webenabled phones expected their mobile Internet use to increase within the next three years [7]. Furthermore, people are changing the ways in which they access information online. According to a recent market research report from the International Data Corporation, by 2015 there will be more people in the US accessing the Internet using their mobile devices than using desktop computers [14].

A recent report from the Association of College and Research Libraries indicates that tight budgets and the use of mobile devices are two key trends for the next few years [2]. These two factors create a situation when library management agrees that the library should provide its content in a mobile-friendly version, which is often difficult to implement due to lack of experience and resources [8]. Some university libraries are able to get their mobile applications as a part of a university-wide mobile application $[15,17]$. However, most academic libraries still have to implement a mobile website or a mobile application by themselves.

To create a mobile-friendly version of library services, one needs to be aware of the different components that make up a typical library website designed for desktop computers. Not all parts of the library website should be made mobile; instead, consider the content which is useful to the mobile user [16]. A recent survey of 22 academic libraries that use mobile websites and/or applications identified a number of common interface features for a mobile library catalog search [17], which include search box and button, search type options, author and title, call number and location, cover image, and status of book.

The features of a desktop library website can be separated into two groups: basic information and online catalog. The basic information of the library web site, such as library hours or programming information, are easier to represent on a mobile device [6]. A mobile library catalog is harder to implement on a mobile device because it is more complex and involves the system's vendor and most libraries' main information system includes an integrated library system (ILS) that uses proprietary software provided by a specific vendor $[3,9]$.

When making a decision about bringing their online services to the mobile devices, libraries have three implementation options: developing a native application, a mobile website, or using a hybrid approach.

There are a number of reasons why a library might choose to develop a native mobile application. The primary reason would be to include the native device functionality, e.g. camera to implement barcode or QR code scanning, compass and accelerometer to determine the orientation of the user, or GPS to determine the user location. Another significant advantage is that some features of the application, e.g. those that do not require a catalog search, may be accessible offline. Creating an application could also increase the exposure to the users by making it available through iTunes App Store or Android Market. Furthermore, some users, especially young people, may consider using an application (as opposed to a mobile website) as a trendier thing to do. Finally, having a native application could be considered as a source of revenue (by charging for the application or by using built-in advertisement), but this is highly unlikely given that libraries are not in the moneymaking business. Native applications offer the advantage of immediate availability of one-click access. Properly designed native applications could be more aesthetically pleasing if they follow the interface convention of their OS platforms.

The drawbacks of using a native application include the extra steps that are necessary to locate and download it onto the device, (but this is needed to be done just once). Developing native applications typically requires a more substantial investment of time and skills. However, our own experience and a number of research reports (e.g. [17]) indicated that it is possible do develop fully functional and well-designed applications by teams of 2-3 graduate students in under 100 hours. This factor is exacerbated further by having to develop a different application for each platform and the diversity of target screen resolutions available on each platform.

Mobile websites are somewhat easier to develop and deploy compared to native applications. The most compelling advantage is that the same mobile website would offer a comparable user experience across all mobile platforms. The biggest drawback is that they require constant data connection. Many user experience studies report that mobile users are less satisfied with mobile website compared with a native application offering the same features. Some of the factors contributing: accessing a website requires several user actions (following one or more links and/or entering a URL), network lag, connectivity issues, etc. A mobile library presence can be implemented as a website using technologies such as HTML, CSS, and JavaScript. The benefit of using a mobile website is compatibility: it will work on every mobile platform that has a standard-compliant web browser.

Hybrid mobile applications could offer a reasonable balance of the advantages offered by both mobile websites and native applications. Like a native application, a hybrid application needs to be developed for the target platform, downloaded and installed on the device, therefore providing the advantage of persistence and instant availability. A hybrid application typically provides a native user interface wrapper around one or more dynamic web pages (or websites) optimized for a mobile platform encompassing the core of the application functionality [16].

To implement a mobile library catalog, one could use a vendor-supplied version of the mobile catalog or an in-house catalog solution [17]. Vendor solutions may be the simplest route to providing a mobile catalog for most libraries. There are some technological issues to be aware of with these mobile catalog options, such as lack of customization, and 
costs. Due to the limitations of the existing vendor-provided options, libraries that want more customization could develop their own mobile catalog solution in-house. This would allow libraries to add any features they want to their mobile library catalog [3].

In a typical library, users often require help on the spot, e.g. where to find books on a particular subject at the library stacks, or how to operate a copying machine. Printed materials, such as posters and placards, are often used to provide such help; however, physical space often provides a number of constraints on their number and size. QR codes offer a solution that solves the space limitation and links the digital content with a physical artifact [1]. QR codes can carry much more information than bar codes enabling them to encode a larger amount of information, e.g. a URL, and, therefore, making it possible to connect the library users to a rich variety of online resources media not limited to text and images, which could include instructional videos and animations, websites for further reference, contacting library staff for help, storing information for further reference, etc. [13]. Consequently, QR codes achieve a double objective by providing more information where it is needed and reduce the visual information overload [12]. QR codes are a very low cost technology that is easy to use and easy to implement.

\section{SYSTEM ARCHITECTURE}

This section outlines a mobile solution created for CCSU library using the Android platform. This system uses the hybrid approach to combine a web application (a mobile website) with the features that can only be provided by a native application. The features of the system can be separated into four logical parts, as shown in Figure 1:

1. Library mobile website content (web),

2. Mobile catalog (web),

3. QR code scanner (native), and
4. Interactive library map (native).

Library mobile website and the mobile catalog are implemented as a web application, in which all application logic is implemented on a web server, while the interactive library map and the QR code scanner are implemented as a native application for the Android platform. To tie the web application and the native application together, we have used a 'Tab layout' interface, as shown in Figure 2.

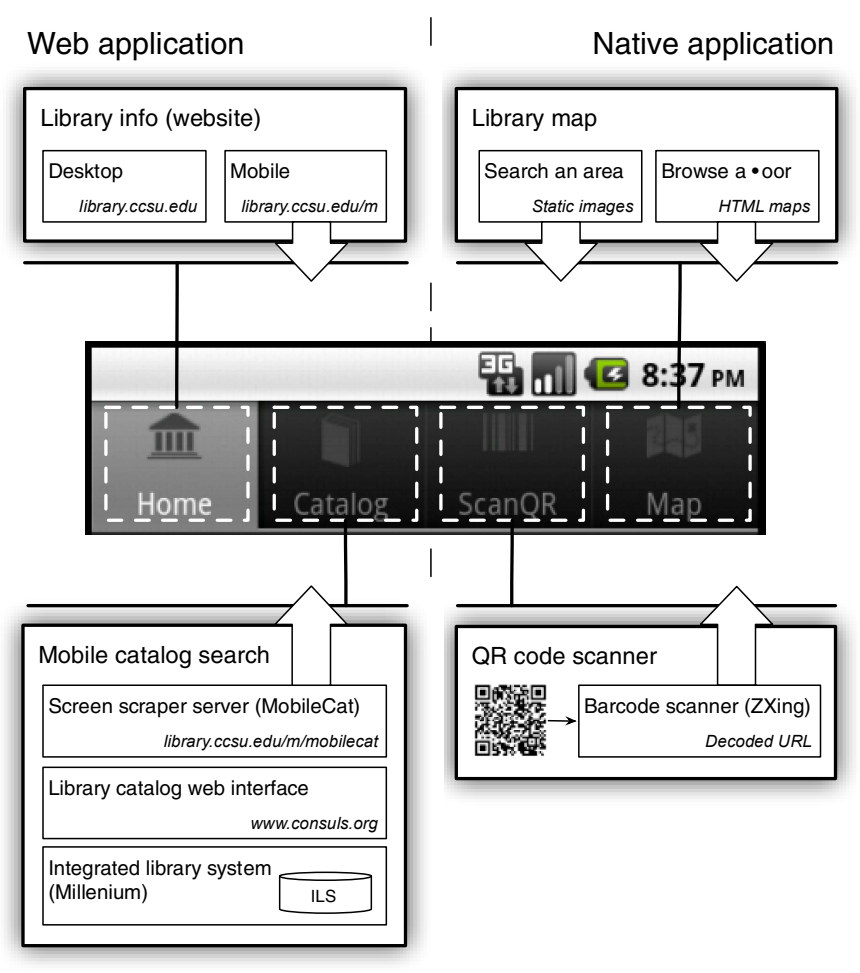

Figure 1. System architecture outline

\begin{tabular}{|c|c|c|c|}
\hline \multicolumn{4}{|c|}{ 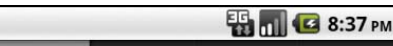 } \\
\hline IIII & [ & IIIII & 15 \\
\hline Home & Catalog & ScanQR & Map \\
\hline \multicolumn{4}{|c|}{ Library Info } \\
\hline \multicolumn{3}{|c|}{$\begin{array}{l}\text { Sunday, 4-1-2012 } \\
2 \mathrm{pm}-9: 45 \mathrm{pm}\end{array}$} & (1) \\
\hline \multicolumn{3}{|c|}{ Contact } & (2) \\
\hline \multicolumn{3}{|l|}{ News } & 8 \\
\hline \multicolumn{4}{|c|}{ Find Articles \& Databases } \\
\hline \multicolumn{3}{|c|}{$\begin{array}{l}\text { Academic Search Premiere } \\
\text { Mobile Friendly Multi-Disciplinary Datab... } \\
\text { Good place to start! }\end{array}$} & 0 \\
\hline \multicolumn{3}{|c|}{$\begin{array}{l}\text { More Databases... } \\
\text { Search by Database Name \& Subjects }\end{array}$} & 0 \\
\hline \multicolumn{4}{|c|}{ Help with Research } \\
\hline \multicolumn{3}{|c|}{ Research Guide } & 0 \\
\hline
\end{tabular}

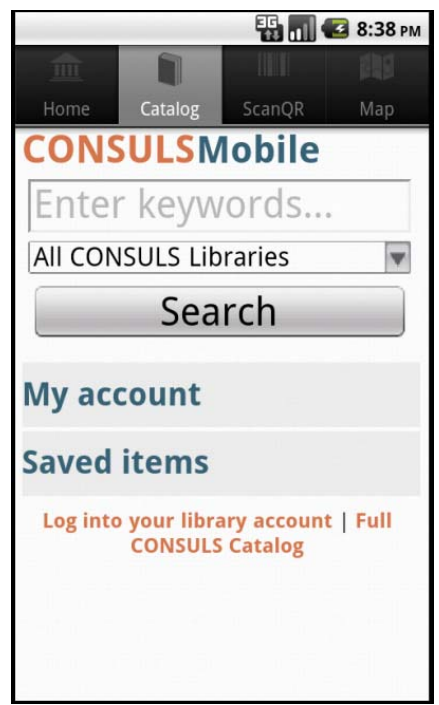

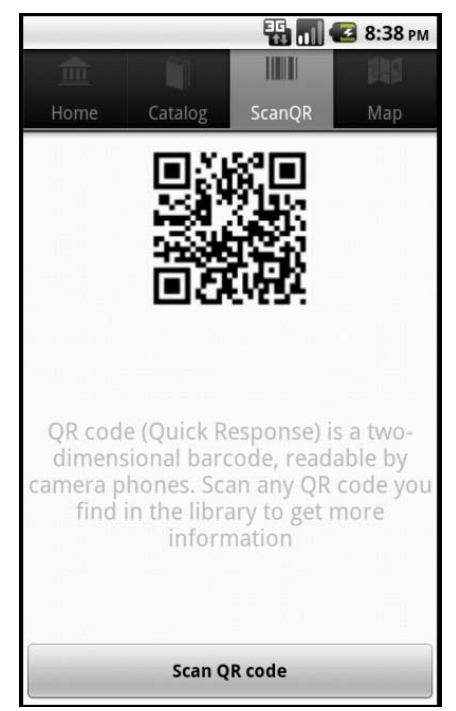

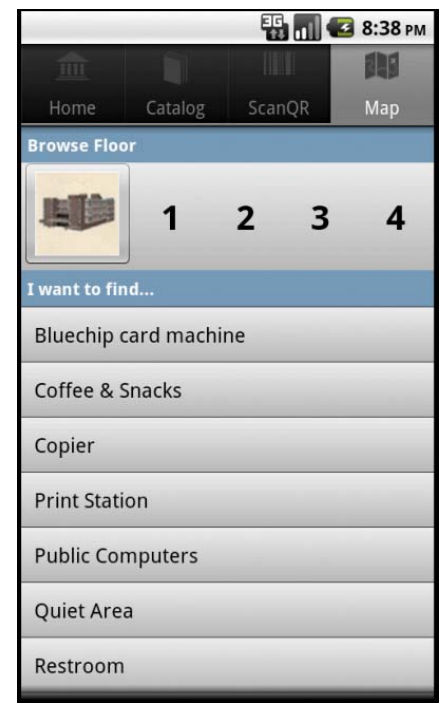

Figure 2. Tabbed layout of the mobile library application. 


\section{A. Library mobile website content}

Library users increasingly use library resources on their mobile devices. However, creating a mobile website cannot be viewed as simply scaling everything down to fit a smaller screen. Most of the time, the users want to complete a simple task or access frequently used information rather than complete a number of complex tasks, such as gathering the entire bibliography from the dissertation. By using web analytic data, we have identified the parts of the library website that were requested most frequently: accessing basic information, such as hours of operation, telephone numbers, or conducting a simple search on the library catalog or databases

As shown in Figure 2, the 'Home' tab contains a web browser control that points to the mobile version of the library website created specifically for this application. The mobile website is implemented using JQueryMobile, and jQuery plugin for mobile web development. This plugin provides all necessary JavaScript and CSS code to display the content properly on mobile devices.

The mobile website is a separate entity from the regular website providing a separate mobile-friendly CSS on every page of the regular website. However, it does retrieve some live information from the regular library website, such as hours of the library and news. Therefore, there is no need to update the content of website in two different locations.

\section{B. Mobile library catalog}

CCSU library is a part of CONSULS consortium (www.consuls.org) that consists of several university and state libraries. The consortium uses Millennium, an integrated library system (ILS) from Innovative Interface Inc. Due to the proprietary nature if this ILS, its database cannot be accessed directly from a third party application. Furthermore, the terms of use prohibit changing the CSS of the library catalog web interface to make it mobile friendly. Therefore, there were three options to create a mobile library catalog. The first option is to acquire an off-the-shelf solution, which is prohibitively expensive at over $\$ 10,000$ a year. The second option is to use the Z39.50 protocol mainly designed for the different ILS to communicate with each other to enable such features as interlibrary loan. This protocol could be used to query the library catalog and receive the results in Machine-Readable Cataloging (MARC) format, which then would have to be converted into XML and displayed on the web as an HTML-formatted page. However, Z39.50 is designed for known-item search (does the library have book A) and not what other items does the library have on subject A. Hence, it does not provide relevancy ranking in the keyword search, which drastically decreases its usability for our purposes. The third solution involves using screen scraping: instead of accessing data directly, it is possible to parse the HTML-formatted search results from the regular catalog and then present them using a mobile-friendly layout.

MobileCat (code.google.com/p/mobilecat) is an open source project for screen scraping the results of Millennium ILS searches. developed at the Tri-College Libraries. MobileCat server acts as an intermediary between a mobile device and the library catalog. The server receives a search query from the mobile device and then passes that query to the library catalog's web interface. The server parses the results returned from the library catalog to add the book cover obtained from Google Books (books.google.com). The server also formats the results with CSS designed specifically for the mobile device.

Map It! function allows the mobile users to identify the stacks location of the items they find in library mobile catalog. It uses the images of the floor plans and stack layouts, as well as the information about the relative physical location of each stack tied to a range of call numbers, as shown in Figure 3. Each search result in the mobile library catalog has a 'Map It!' button that uses an Android SDK functionality to bind JavaScript code with a native application.

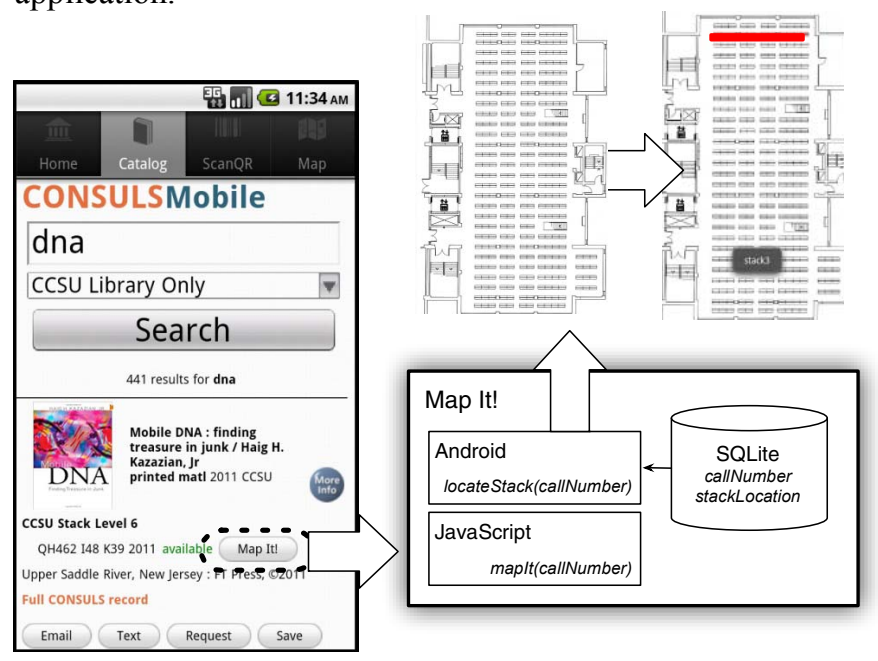

Figure 3. Operation of the Map it! function.

\section{QR code scanner}

Our system incorporates a $\mathrm{QR}$ scanner to link physical objects in the library with the relevant digital content. For example, we placed a $\mathrm{QR}$ code next to a microfilm reader machine linking it to an online video explaining how to use the machine. The system uses Zebra Crossing (code.google.com/p/zxing), an open-source multi-format $1 \mathrm{D} / 2 \mathrm{D}$ bar code image processing library implemented in Java. To generate QR codes, we used Google Chart Tools (code.google.com/apis/chart).

\section{Interactive library map}

Mobile devices allow the building manager to replace clunky paper maps with electronic maps. Electronic maps are not only more portable and interactive but also provide many added features. For example, users could tap an area of interest on the map and the system would show more information about that area. The system can also automatically locate an area that the user wants to go to and display that area on the map.

The map function is implemented using the Android WebView object in order to utilize its built-in zoom and pan functions. Therefore, this functionality is basically a series of 
web pages that are stored locally inside the application. The user does not need an Internet connection to use this function.

The user can select an area of the library they want to find. To display a specific area such as a restroom or copier, the system simply displays a static image file that was created specifically for that area. The user can also browse each floor on the library, which allows the user to interact with the map by tapping on a selected area to get more information. This function is implemented using HTML image maps. When the user taps on the area, the JavaScript bind function shows the native popup dialog with additional information about that area.

\section{SYSTEM EVALUATION}

Once the system was developed, a number of users were recruited to test its usability and provide feedback to the developers. Our objective was to find out how well our existing library mobile application performs within the mobile environment. During the testing, we asked ten participants to use this mobile application to complete set of tasks on actual Android devices, varying from mobile phones to tablets. Upon the completion of the test, the study participants are asked to complete a questionnaire to survey the application's usability.

Testers could use their own phone if they preferred, but for those who did not have an Android device, a 7-inch Android tablet had been given for the test and a 2.8-inch Android phone had been given for the QR code function (since the tablet doesn't have a camera). Prior to the test, minimal information about the application had been given to the testers. Only one participant who did not have a prior experience using the Android operating system had been briefly introduced to the basic operation of the phone.

\section{A. Task Completion}

Tasks 1-3 were designed to identify the usability of the home tab function, which provides users with the basic information about the library, such as hours, contact, and research help. Tasks 4-6 were designed to test the usability of the Catalog, Library Map, and QR code tabs by asking the testers to search for a book using the mobile library catalog and then retrieve it from the stack using the MapIt! function as a guide, locate frequently used areas in the library such as the reference desk and quiet study areas, and to scan a QR to find out how to use the microfilm machine.

Task 1: Find library hours. All testers were able complete this task quickly because the current hours of the library are prominently presented first on the list, so users noticed it instantaneously. All testers had selected 'hour' to see detailed hours.

Task 2: Contact reference librarian via instant messaging chat. All participants were able complete this task. One of the testers needed a hint that it is possible to scroll down to see more options. Most of the subjects $(80 \%)$ went directly to 'Contact Reference Librarian', while $20 \%$ went to 'contact' first, which only shows the telephone number of the reference desk.
Task 3: Accessing research guide. LibGuide is a content management system, which the library uses to host a multitude of research guides. The system allows every librarian to easily create and edit content without knowledge of the HTML language. Users could complete the task of finding a guide about citing resources by going to 'Research Guide'. When they reached the LibGuide interface, they could find the guide they needed by either using the search bar, or using the browse function that allows them to browse guides by popularity, recent, and subject. Seven out of ten participants completed this task. All of them went to the right place, 'research guide' and they used either search or browse guide function to find the guide they were looking for. But the testers who failed the task seemed confused and distracted by the layout of LibGuide interface. Hence, they did not notice that they could find the guide by using either search or browse function. A major distraction came from the feature guide item (Adventure in Research Podcast) that takes a majority of the screen and makes browse and search guide a lot less prominent. Also, some users who failed the task did select browse by subject function, but did not go through the whole list to find the guide about citing resources.

Task 4: Finding item/area in the library using the Map function. To access the library map function, a user needs to select the tab on top of the application interface. Since the application starts in the 'Home' tab by default, three test subjects showed difficulty recognizing this because they did not notice that the application consisted of four tabs that they can select. Test conductor had given a hint about the tab layout in order for them to find the map function.

Once the participants get to the map tab, finding restrooms and quiet areas is very obvious. Only one participant who failed this task was having trouble with the touch interface. He/she knew what item in the list to select but the system misinterpreted the input, causing the application to show a wrong map.

Apart from allowing the users to find a particular area, the map function also allows users to browse the maps of every floor of the library. When a user is in the 'browse floor' mode, an area of interest will be marked with an icon and the area is highlighted. This indicates that the area is interactive; user can select it to find out information.

Task 5: find specific books using the mobile catalog. Participants were asked to find two books. First, in a known item search, the user was given a specific title. Second, the user had to find a book about a specific subject. There were also sub tasks that asked them to save books for later and to retrieve a book from the stacks using the 'Map It!' book locator function.

All testers were able to complete both of the tasks. The first task, find a specific title, was easier to complete. Most testers were able to find the book with single query. One tester conducted the search using author name as a keyword for which library catalog returned a correct result.

The second task was to find a book about a particular subject; all testers were able to complete this task, but with less ease. None of the testers were able to find the right book with their first keyword. They needed to refine their keyword 
and performed one or two more queries to find the book they wanted. This problem is largely caused by the ranking algorithm of the library catalog which is not as advanced as a commercial Internet search engine such as Google which users are more familiar with. One example of queries that return an unsatisfactory result is 'reclassification of Pluto' ( 0 result).

When the user locates the book they want, they can tap on it to expand view option. When the view is expanded, the user will be able to access the 'save' button to save the book for later use along with other buttons, such as e-mail and text book info and call number to themselves, and request the book from other CSU campuses. $90 \%$ of the testers were able to complete this task by themselves. Only $10 \%$ of the testers needed a hint that they can tap on the book to expand to see other options. Once the book is saved, testers were asked to locate their saved items, and use the 'Map It!' function to display an approximate location of the book on the stack.

Task 6: Scan a QR code. Testers were given a simulated situation where they are trying to use the microfilm machine but they don't know how to load the film into the machine. A poster with a QR code was given to the testers to scan in order to learn how to use the machine. When the user scans the QR code, the application shows a video of how to load the film into the machine. The task completion rate is very satisfactory. Most of the users were able to complete the tasks by themselves $(70 \%)$ while $20 \%$ of users needed help to complete the task and only $10 \%$ of testers failed to complete the task.

\section{B. Usability of the application from questionnaire}

After completing the test, the users were asked to complete a questionnaire. The questionnaire consisted of 30 questions with possible scores ranging from 1 to 7 from strongly disagree to strong agree. Also there were three more open-ended questions asking about suggestions in general.

A total of 30 questions consisting of 6 sets of questions (5 each) were designed to gauge the users' satisfaction with each sub-function of the application (home tab, mobile catalog, Map It!, QR code scanner, Library map, and the application overall). The results are summarized in Figure 4.

The users strongly agreed that the information about library presented in home tab is useful, especially, library hours and ability to chat with reference librarian. However, users are not sure that the content available in the home tab is relevant enough for a mobile user. Testers think the mobile library catalog is the most useful feature of the application. The users responded very positively about searching the library catalog on mobile phone and they especially like the save-for-later function. Working in conjunction with the mobile library catalog is the Map It! Function, testers thought the Map It! locator is helpful in finding books and it makes it easier to find the book on the stack. However they wish the location of item highlighted in the floor map could be more specific.

The users felt positive, in general, about the QR code scanner function. They think the QR code scanner is easy to use and useful. However since the $\mathrm{QR}$ code reader built into the application has no value-added function that would differentiate it from other QR code scanners, the users were somewhat neutral if QR code scanner in our application is any more useful than other application they can download.

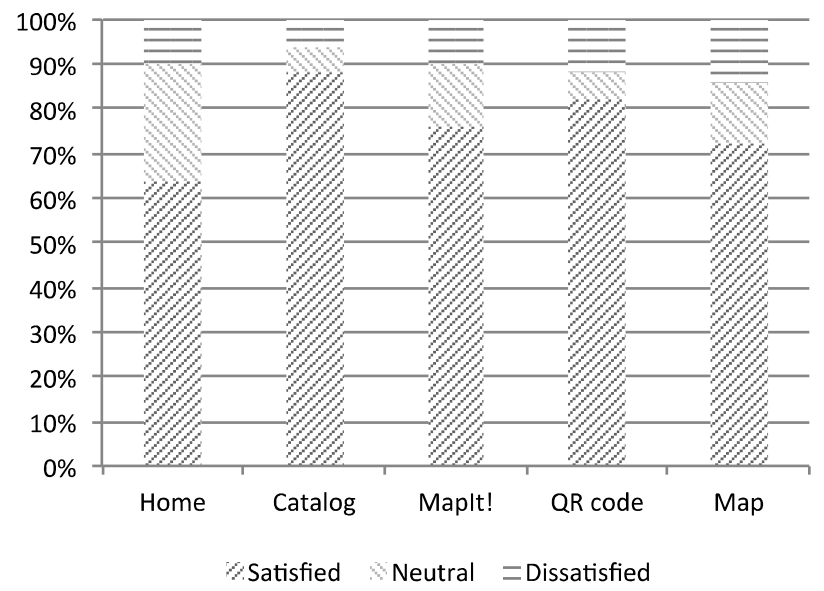

Figure 4. User satisfaction with the features of the system.

The testers liked the library map function in general. They think the map is useful for them in navigating the library building to find items or areas in the library. However it is unclear how practical this function is. Users answered neutrally about the time it takes to use map function to locate an area in the library might be longer than just looking at physical signage or asking someone.

Overall, the users were satisfied with the application. They think it is useful, not hard to use, and would likely install it on their Android devices.

\section{Elaboration}

The questionnaire concluded with three open-ended questions asking the users about the areas of the application's functionality the testers thinks is the most useful, additional features they would like to see, and whether there is anything they would like to change in this application.

The most useful function, consistent with the questionnaire results, is the mobile catalog search. For additional features, the users would like to see more advanced functions such as the ability to scan a QR code from the desktop version of the library catalog resulting in the mobile application automatically saving the book information on the phone instead of the user writing it down on paper manually; an ability to preview the content of books, and the search term suggestion much like Google's auto complete.

One of the major changes the users would like to have is to fix the resolving the issues of saving the book information in the mobile library catalog. The users want to be able to access the information on their mobile device when there is no Internet connection. 


\section{SUMMARY}

Mobile application described in this paper fulfills the need of the Android device users to access library data anytime anywhere on their mobile devices. Our user studies indicate that $72 \%$ to $75 \%$ of the testers liked all the features built into this mobile application.

Based on the usability testing of this mobile application, we identified two areas of functionality in need improvement. Currently, the user has the ability to save the book information they were searching for in the mobile catalog using this mobile application. But if the user is not connected to Internet, they cannot access this information. In the next release of this mobile application, we will implement this save function using a local database allowing the user to access their saved items without the network connectivity.

The current version of the mobile application has the map function divided in two parts, a static map and a dynamic map. The Map it! Function on the catalog page is connected with the local database inside the application containing stacks location information along with the corresponding ranges of call numbers. In order to enable the dynamic map functionality, it is the responsibility of the administrator of the application to change the coordination of book call numbers relative to the stacks locations inside the database. The static maps, such as floor details, copier and printer maps, are actually embedded in the image of each floor. So, if the position on the printer is changed in the future, then the administrator has to change the image itself, which is rather cumbersome. The next version of this mobile application all static map functionality will be replaced with dynamic, eliminating the need for embedding any information about areas of interest (location of printers, reference desk, etc.) into the image itself.

Another area of improvement will be user interface of the Map it! Function. In the current version of the mobile application, when the user searches the book, it shows the detail of the book location, such as the title and call number of the book. After that, when the user uses the Map it! Function, it displays the map and the location of the book they were looking for. The user cannot see the details of the book location of the map. In the next release of the library mobile application, we will improve the user interface design so that the user can see the map and the details of the book location information on the same screen.

\section{REFERENCES}

[1] R. Ashford, "QR codes and academic libraries: reaching mobile users," College \& Research Libraries News, Vol. 71, No. 10, pp. 526530 , Nov 2010.

[2] ACRL Research Planning and Review Committee, "2010 top ten trends in academic libraries," College \& Research Libraries News, Vol. 71, No 6, pp. 286-292, June 2010.

[3] L. Bridges, H. G. Rempel, K. Griggs, K. "Making the case for a fully mobile library web site: from floor maps to the catalog," Reference Services Review, Vol. 38, No. 2, pp. 309-320, 2010.

[4] K. Church, N. Oliver, "Understanding mobile web and mobile search use in today's dynamic mobile landscape," $13^{\text {th }}$ International Conference on Human Computer Interaction with Mobile Devices and Services (MobileHCI '11), pp. 67-76, Aug-Sep 2011.

[5] K. Church, B. Smyth, P. Cotter, K. Bradley, "Mobile Information Access: A Study of Emerging Search Behaviour on the Mobile Internet," ACM Transactions on the Web, Vol. 1, No. 1, May 2007.

[6] M. Connolly, T. Cosgrave, B. Krkoska, "Mobilizing the Library's Web Presence and Services: A Student-Library Collaboration to Create the Library's Mobile Site and iPhone Application," Reference Librarian, Vol. 52, No. 1-2, pp. 27-35, 2011.

[7] EDUCAUSE, "The ECAR study of undergraduate students and information technology," available at www.educause.edu/ers1006, 2009

[8] R. T. Greenall, "Mobiles in Libraries," Online Magazine, Vol. 34, No. 2, pp. 16-19, 2010.

[9] C. W. Hanson, "Mobile solutions for your library," Library Technology Reports, Vol. 47, No. 2, pp. 24-31, Feb/Mar 2011.

[10] International Telecommunication Union, "Information society statistical profiles 2009: Americas," available at www.itu.int/dms_pub/itu-d/opb/ind/D-IND-RPM.AM-2009-E09PDF-E.pdf, 2009.

[11] A. Kaikkonen, "Full or tailored mobile web - where and how do people browse on their mobiles?" International Conference on Mobile Technology, Applications, and Systems, Sep 2008.

[12] D. Pons, R. Vallés, M. Abarca, F. Rubio, "QR codes in use: the experience at the UPV Library," Serials: The Journal for the Serials Community, Vol. 24, No. 3, pp. S47-S56, Nov 2011.

[13] A. Walsh, "QR Codes - using mobile phones to deliver library instruction and help at the point of need," Journal of Information Literacy, Vol. 4, No. 1, pp. 55-63, 2010.

[14] K. Weide, "IDC: More Mobile Internet Users Than Wireline Users in the U.S. by 2015," International Data Corporation, retrieved from http://www.idc.com/getdoc.jsp?containerId=prUS23028711

[15] S. Wilson, G. McCarthy, "The mobile university: from the library to the campus," Reference Services Review, Vol. 38, No. 2, pp. 214232,2010 .

[16] J. Wisniewski, "Mobile that works for your library," Online Magazine, Vol. 35, No. 1, pp. 54-57, Jan/Feb 2011.

[17] Y. Zhou, R. Broussard, M. Lease. "Mobile options for online public access catalogs," 2011 iConference, pp. 598-605, Feb 2011. 